\title{
UCRL-JRNL-222899
}

LAWRENCE LIVERMORE N A TION A L LABORATORY

\section{Ultrasensitive Searches for the Axion}

K. A. van Bibber, L. J. Rosenberg

July 17, 2006

Physics Today 
This document was prepared as an account of work sponsored by an agency of the United States Government. Neither the United States Government nor the University of California nor any of their employees, makes any warranty, express or implied, or assumes any legal liability or responsibility for the accuracy, completeness, or usefulness of any information, apparatus, product, or process disclosed, or represents that its use would not infringe privately owned rights. Reference herein to any specific commercial product, process, or service by trade name, trademark, manufacturer, or otherwise, does not necessarily constitute or imply its endorsement, recommendation, or favoring by the United States Government or the University of California. The views and opinions of authors expressed herein do not necessarily state or reflect those of the United States Government or the University of California, and shall not be used for advertising or product endorsement purposes. 


\title{
Ultrasensitive Searches for the Axion
}

\author{
Karl van Bibber and Leslie J Rosenberg
}

The axion, a hypothetical elementary particle arising from a compelling solution to the strong-CP problem, has eluded discovery for three decades. Experiments based on coherent axion-photon mixing in strong magnetic fields are just now reaching the sensitivity to detect it, either as the dark matter or as a component of the solar flux. Although of lower sensitivity, purely laboratory experiments hold potential for surprise.

Fifty years ago, Norman Ramsey and collaborators endeavored to measure, for the first time, the electric dipole moment of the neutron. What they measured, to very high precision, was consistent with zero: $\left|d_{n}\right|<5 \times 10^{-20} \mathrm{e} \cdot \mathrm{cm}$. In the halfcentury since then, the neutron e.d.m. has become of the most remarkable precision measurements in modern physics. Currently the limit has been improved to a vanishingly small $10^{-26} \mathrm{e} \cdot \mathrm{cm}$; experiments being readied may reach $10^{-28} \mathrm{e} \cdot \mathrm{cm}$. This result is puzzling since within the Standard Model, the strong interaction should be $\mathrm{T}$ - and thus $\mathrm{CP}$-violating, implying a large electric dipole moment ten orders of magnitude larger than the current bound. In 1977, two young theorists at SLAC, Roberto Peccei and Helen Quinn discovered a simple dynamical mechanism to enforce strong-CP symmetry [1], which as Steven Weinberg and Frank Wilczek independently realized, implied the existence of a new elementary particle, termed the axion [2].

The axion is a pseudoscalar, meaning that it has the quantum numbers of neutral pion. Its mass and all its couplings are inversely proportional to the one key parameter of the Peccei-Quinn theory, a symmetry-breaking scale $f_{a}$, whose 
value is unknown, other than it must be very large. (A popular, if somewhat fanciful account of how the axion arises in particle physics is found in the December, 1996 Physics Today article "The Pool-Table Analogy of the Axion" [3].) Peccei and Quinn originally assumed, for no good reason, the symmetrybreaking to occur at the electroweak scale, i.e. $\mathrm{f}_{\mathrm{a}} \sim \mathrm{f}_{\mathrm{EW}}$, resulting in axions of $\sim$ $100 \mathrm{keV}$ mass, and coupling strongly enough to have been seen in acceleratorbased experiments. Such axions were quickly ruled out, but theorists found how to construct axion models with much higher values of $f_{a}$. As a consequence, its mass can be no more than an electronvolt, and likely less than a millielectronvolt. Its interactions with matter and radiation were driven down proportionately, to extraordinarily feeble levels, many orders below that of the weak scale.

\section{Axion-photon mixing.}

Nevertheless, over the past two decades, several experiments around the world have begun to approach sensitivities required for detection of cosmological or astrophysical axions. The axion in general couples widely across the particle spectrum. It necessarily possesses fundamental ("tree level") couplings to quarks, and optionally to leptons; and it has induced couplings to nucleons and photons. Interestingly however, all of the current searches look through the axion-photon interaction. Why this is so bears a little discussion.

Like the $\pi^{0}$, the axion possesses a two-photon interaction; also like the neutral pion, one of the photons may be virtual. Specifically, a photon can interact in a classical electromagnetic field producing a pseudoscalar, and vice versa. As an historical interlude, the first accurate analysis of the neutral pion lifetime $\left(\sim 10^{-16}\right.$ s) was made by Henry Primakoff in 1951, who realized that the measurement of the $\pi^{0}$ lifetime was synonymous with the vastly easier measurement of its photoproduction cross section in the Coulomb field of a high- $Z$ nucleus, as they are both determined by the same vertex $\mathrm{g}_{\pi \gamma \gamma}$. Beginning in the 1980's, several theoretical calculations and proposals to search for the axion based on the 
Primakoff effect appeared, with two common motifs. First, the coherent mixing of the axion and photon states over large spatial regions of high magnetic field could result in measurable conversion rates, making up for the exceedingly small coupling $g_{\alpha \gamma \gamma}$. Second, the advent of large high-field superconducting magnets provided the technological basis for these proposals. Experiments promptly ensued, all variations on the same theme.

\section{Axionic dark matter - "tuning for radio KSVZ"}

The axion also fit the bill as an ideal candidate to comprise the five-sixths of all matter in the Universe that is concluded to be non-baryonic. It would be produced as a zero-temperature Bose gas during the Big Bang, never be in thermal equilibrium with the rest of the Universe, and its lifetime would be so great as to be effectively stable. Most importantly, sufficiently light axions would dominate the cosmological energy density budget. In fact, axions lighter than a micro-electronvolt would have severely overclosed the Universe, whereas axions heavier than a milli-electronvolt produced in SN1987a would have dramatically foreshortened its neutrino pulse. Neither being the case, the two arguments together constitute the prime axion mass window, $\sim 10^{-(6-3)} \mathrm{eV}$.

In 1983, Pierre Sikivie proposed an elegant and ingenious experiment to detect dark matter axions by their resonant conversion to RF photons in a microwave cavity permeated by a strong magnetic field [4] (Figure 1). Tuning the cavity to fulfill the resonant condition, $\mathrm{h} v=\mathrm{m}_{\mathrm{a}} \mathrm{c}^{2}\left(1+O\left(\beta^{2} \sim 10^{-6}\right)\right)$, and assuming axions saturate the galactic halo, the conversion power from an optimized experiment is given by:

$$
P(a \rightarrow \gamma) \approx\left(10^{-23} W\right) \cdot B[T]^{2} V\left[m^{3}\right] Q\left[10^{5}\right] \cdot m_{a}[\mu e V] g_{\gamma}^{2}
$$

where $B$ is the strength of the magnetic field, $V$ the cavity volume, $Q$ the cavity quality factor, and $g_{\gamma}$ is the dimensionless axion-photon coupling, of order unity 
for a wide range of models. The expected axion signal is exceedingly tiny, measured in yoctowatts $\left(10^{-24} \mathrm{~W}\right)$, the smallest SI unit of power. In comparison, in 2002 the last signal ever received from the 7.5-watt transmitter aboard Pioneer 10, then 12.1 billion kilometers from Earth, was a prodigious $2.5 \times 10^{-21} \mathrm{~W}-$ and unlike the axion, we knew its frequency! Fortunately, the photon coupling of very different axion models cluster in a tight band, thus bounding its parameter space not only in $\mathrm{m}_{\mathrm{a}}$, but also in $\mathrm{g}_{\gamma}$. Various models historically have served as goal-posts for experimentalists. Half-way through the model band, corresponding to one implementation of a general class of axions, is denoted KSVZ (for Kim-Shifman-Vainshtein-Zhakarov); at the lower edge of the model band is the most pessimistic model, the GUT-inspired DFSZ (for Dine-FischlerSrednicki-Zhitnitskii).

In the late-1980s, pioneering experiments of only a few liters volume, one by a Rochester-Brookhaven-Fermilab collaboration and the other by the University of Florida already achieved power sensitivities within a factor of 100-1000 of the model band over a narrow range in frequency [5,6] (Figure 2).

In an actual search however, signal power is one of only three factors an experimenter can control. The ultimate signal-to-noise ratio, and hence detectability of any signal is governed by the Dicke radiometer equation,

$$
\frac{s}{n}=\frac{P_{s}}{P_{n}} \sqrt{t \cdot \Delta v}=\frac{P_{s}}{k T_{n}} \sqrt{\frac{t}{\Delta v}}
$$

where $\mathrm{s} / \mathrm{n}$ is the signal-to-noise ratio, $\Delta v$ the bandwidth of the signal, $\mathrm{t}$ the integration time, and $\mathrm{P}_{\mathrm{S}}$ and $\mathrm{P}_{\mathrm{n}}$ the signal and noise power respectively. There are practical limits to increasing either the signal power (the size and cost of superconducting magnets), or the integration time at each frequency (the need to scan decades in frequency within a finite number of years). This puts a clear premium on reducing the total system noise temperature, which is the sum of the physical temperature and the equivalent electronic noise temperature of the 
amplifier, $T_{n}=T_{\text {phys }}+T_{\text {elec }}$. Two second-generation experiments were designed to finally achieve sensitivity to halo axions, both by scaling up in volume, but more importantly driving the technology of ultra-low noise microwave detection. These efforts were complementary, even in the quantum sense - the experiment at Livermore (LLNL) exploits the character of photon-aswave, while the other at Kyoto has gone the photon-as-particle route.

In its first phase of operation, now concluded, the Axion Dark Matter eXperiment $^{*}$ (ADMX) at LLNL used heterojunction field-effect transistor (HFET) amplifiers to listen for the axion-photon conversion in a microwave cavity. These amplifiers represented a major leap in ultra-low noise amplifier technology when introduced into common use in the 1970s, and this experiment benefited from the long-term development program at NRAO which delivered packaged amplifiers of ever improving noise performance for the radio astronomy community [7]. In these devices, electrons from the $\mathrm{Al}_{\mathrm{x}} \mathrm{Ga}_{1-\mathrm{x}} \mathrm{As}$ donor layer fall into the GaAs 2D quantum well, which serves as the FET channel. Separating the function of the donor, whose own donor atoms represent scattering centers, from the undoped channel, results in essentially ballistic electron transport, thereby minimizing scattering in the channel and thus minimizing electronic noise. These amplifiers currently provide the world's best broadband noise performance in the gigahertz range $(\sim 1.5 \mathrm{~K})$, and enabled the exclusion of KSVZ axions over an octave in mass (Figure 2) [8].

The best HFET amplifiers nevertheless are still far noisier than the quantum limit, $\mathrm{T}_{\text {quant }}=\mathrm{hv} / \mathrm{k} \sim 50 \mathrm{mK}$ at $1 \mathrm{GHz}$. An amplifier approaching the quantum-limit would dramatically improve both the sensitivity and search rate of the axion experiment. In 1996, John Clarke and coworkers at Berkeley developed a new amplifier based on microstrip-coupled dc SQUID (Figure 3, left); high gain and very low noise have been demonstrated in a narrow bandwidth around the microstrip's resonant frequency, well into the gigahertz range [9]. Unlike the complicated noise behavior of heterojunction transistor amplifiers as the temperature is lowered, the intrinsic noise of the SQUID is proportional to the physical temperature, i.e. $\mathrm{T}_{\text {elec }} \sim \mathrm{T}_{\text {phys }}$, the origin being the 
Johnson noise in shunt resistors across the Josephson junctions. The expected noise behavior is observed as the device is cooled (Figure 3, right), until flattening out within $50 \%$ of the quantum limit. Newer SQUID designs incorporating micro-cooling fins on the shunt to enhance coupling of athermal electrons to the lattice have edged closer yet to the quantum limit.

A phased upgrade of ADMX with SQUID amplifiers began in mid-2004 supported by the U.S. Department of Energy, with initial operation expected in late-2006. Ultimately, the retrofitted experiment will be capable of detecting DFSZ axions, even comprising only a fraction of the halo, and scanning much faster. In addition to their dark-matter application, these early near-quantumlimited devices have already found their way into several other experiments around the world. In particular they have been coupled with single-electron transistors (SETs) for qubit readout in quantum computation schemes.

Seishi Matsuki and collaborators in Japan, have pursued an alternative detection scheme for their axion search, which in principal can entirely evade the quantum limit. In the early 1970's Dan Kleppner and others realized that Rydberg atoms, those for which a single electron has been promoted to a level with a large principal quantum number $\mathrm{n}$, could serve as a single-quantum microwave detector, effectively a noise-free "RF photomultiplier tube". As their energy spectrum is quasi-hydrogenic, i.e.

$$
E_{n, l}=\frac{R}{\left(n-\delta_{l}\right)^{2}} \equiv \frac{\Re}{\tilde{n}^{2}}
$$

where $\mathscr{R}$ is the Rydberg constant and $\delta_{1}$ an angular momentum-dependent correction factor, available dipole transitions $|n, 1\rangle \rightarrow|n, 1 \pm 1\rangle$ can be found anywhere in the microwave spectrum by the appropriate choice of $n$, and the transition energy can then be Stark-tuned to produce an exact match to a desired frequency. Furthermore, Rydberg atoms possess both very large dipole 
transition probabilities and suitably long lifetimes, both required for practical microwave detectors in a cavity-atom geometry.

In their experiment, the conversion cavity is coupled to a second, detection cavity tuned to the same resonant frequency $v$ (Figure 4, left). A beam of Rydberg atoms is prepared by two-step laser excitation, e.g. from the ground state of rubidium to a $\mathrm{ns}_{1 / 2}$ state. The beam then traverses the detection cavity, where the spacing of the $|n p\rangle$ and $|n s\rangle$ levels is Stark-adjusted to equal hv, resulting in a coupled atom-cavity-photon system with high efficiency for photon absorption. Finally, the mixed-population beam exiting the cavity undergoes selective field ionization [10]. Being a phaseless measurement, the Rydbergatom technique is not subject to the standard quantum limit itself, but there are other noise sources that must be mitigated in practice. The Kyoto experiment is designed with the ultimate goal of $\sim 10 \mathrm{mK}$ noise temperature, dominated by the cavity blackbody spectrum, if all other contributions can be suitably tamed. The small volume CARRACK-I experiment has measured blackbody photons in the cavity down to $60 \mathrm{mK}$ (Figure 4, right) [11], and has completed a scan of $8 \%$ in mass range around $10 \mu \mathrm{eV}$. Sensitivities far better than DFSZ are possible from the scaled-up CARRACK-II.

What is sacrificed with Rydberg-atom detection, not being a spectral receiver, is the ability to detect structure narrower than the bandpass of the cavity, i.e. $\Delta \mathrm{E} / \mathrm{E}$ $\sim 10^{-5}$. By contrast, ADMX has implemented a high-resolution channel which is capable of seeing energy-spectrum fine-structure in the axion signal down to $\Delta \mathrm{E} / \mathrm{E} \sim 10^{-11}$, as predicted in certain models of galactic halo formation.

\section{Searches for solar axions}

It was also realized early on that axions could be produced thermally in stellar burning and escape without subsequent scattering; and even if this happens only very rarely, it would represent a highly efficient mechanism for energy transport out of the star. The consequences for stellar evolution would be strikingly apparent if the axionic luminosity were to become comparable to the photon 
luminosity in the case of main sequence stars, or to the neutrino luminosity in the case of Type-II supernovae, such as SN1987a. Setting limits on particle physics exotica, such as axions, by the concordance between observed and calculated stellar evolution thus became a powerful tool in establishing limits on the couplings of hypothetical elementary particles, far more restrictive than accelerator-based experiments [12]. In general, introducing a channel for freestreaming energy loss from a star's core accelerates its evolution, as it will contract and heat up under the influence of gravity when axions (or whatever) compete with the production of strongly-trapped photons, which act to counterbalance gravitational pressure. Furthermore, for each stellar system, axions are excluded only over a finite range of couplings. As the axion's coupling is dialed up in the transport codes, the free-streaming lower limit is first reached, where deviations from the model without axions becomes noticeable. Eventually however, as the coupling is further increased, the axions themselves become strongly trapped; the upper limit corresponds to where their influence on evolution diminishes below threshold of observation.

It is remarkable that in the same paper where Sikivie outlined how dark-matter axions could be detected by their coherent conversion to microwave photons, he further invented the technique for detection of axions streaming from the Sun's core [4]. In this 'axion helioscope', a long dipole magnet is pointed towards the Sun, with an x-ray detector at the back end. The solar production of axions is dominated by the Primakoff process $\gamma+\mathrm{Ze} \rightarrow \mathrm{a}+\mathrm{Ze}$; and their spectrum, and thus that of the detected photons is roughly Maxwellian with a mean energy $\left\langle E_{a}\right\rangle$ $=4.2 \mathrm{keV}$ (Figure 5, upper left). That this is higher than the core temperature of $1.3 \mathrm{keV}$, is due to plasma screening effects which suppress the production at lower energies. The integrated solar flux at the Earth is given by $\Phi_{\mathrm{a}}=7.44 \times$ $10^{11} \mathrm{~m}_{\mathrm{a}}^{2}[\mathrm{eV}] \mathrm{cm}^{-2} \mathrm{sec}^{-1}$ (for KSVZ axions). The upshot of ref. [4] is that the direct detection of axions could ultimately improve on limits from inferences from solar evolution, and would in any case prove much more reliable. The integrated rate of detected x-rays is given by Equation (4) 


$$
\begin{aligned}
& \mathrm{R}_{\mathrm{x}}\left[\mathrm{sec}^{-1}\right]= 3.68 \times 10^{-7} \mathrm{~A}\left[\mathrm{~cm}^{2}\right] \cdot \mathrm{B}^{2}[\mathrm{~T}] \cdot \mathrm{L}^{2}[\mathrm{~m}] \cdot \mathrm{m}_{\mathrm{a}}^{2}[\mathrm{eV}] \cdot \mathrm{F}^{2}(\mathrm{q}), \\
& \text { with } \mathrm{F}(\mathrm{q})=\int \mathrm{dx} \mathrm{e}^{-\mathrm{iqx}} \mathrm{B}(\mathrm{x}) / \mathrm{B}_{0} \mathrm{~L}
\end{aligned}
$$

where $\mathrm{B}$ is the strength of the magnetic field, $\mathrm{L}$ its length, and $\mathrm{A}$ the detection area. F(q) represents the 'form-factor' of the magnetic field with respect to the momentum mismatch between the massive axion and massless photon of the same energy, $\mathrm{q}=\mathrm{k}_{\mathrm{a}}-\mathrm{k}_{\gamma}=\left(\omega^{2}-\mathrm{m}_{\mathrm{a}}{ }^{2}\right)^{1 / 2}-\omega \sim \mathrm{m}_{\mathrm{a}}{ }^{2} / 2 \omega$. This momentum mismatch defines the oscillation length in the problem, $1=2 \pi / q$, where the axions are no longer sufficiently relativistic to stay in phase with the photon for maximum mixing, and the conversion probability begins to roll off. High energy accelerator dipoles are ideal for this application; the CAST (CERN Axion Search Telescope) experiment utilizes a prototype LHC dipole magnet on a movable mount to track the Sun for several hours at dawn and dusk. Possessing two separate magnet bores, both apertures on each end can be instrumented. The CAST collaboration have recently published the best limits on the solar axions, equaling those derived from horizontal branch stars [13] (Figure 5, upper right).

In the ongoing Phase II run, CAST will push the sensitivity of the search upward in mass into the region of axion models, by introducing a gas $\left({ }^{4} \mathrm{He}\right.$ and later $\left.{ }^{3} \mathrm{He}\right)$ of variable pressure into the magnet bore. The plasma frequency $\omega_{\mathrm{p}}=$ $\left(4 \pi \alpha N_{e} / m_{e}\right)^{1 / 2} \equiv m_{\gamma}$ endows the $x$-ray photon with an effective mass; thus full coherence of the axion and photon states can be restored, and the theoretical maximum conversion probability achieved for any axion mass, by the filling the magnet with a gas of the appropriate density [14]. The mass range can thereby be extended upwards in scanning mode, by tuning the gas pressure in small steps to as high as feasible. Probing the electronvolt range directly by the solar experiment is certainly very worthwhile, as the closure between the upper end of the SN1987a bound and the lower end of the red giant bound is incomplete at best. In fact, the situation of the observed neutrino signals from SN1987a from 
Kamiokande and IMB has always been problematic in detail, which must correspondingly temper one's confidence in any limits on exotica so derived.

\section{Purely laboratory experiments}

Sensitive axion searches based on axion-photon mixing can also be carried out without resort to either cosmological or astrophysical sources, and all their attendant uncertainties, although the sensitivity achieved to date by purely laboratory experiments are many orders of magnitude removed from the expected coupling of QCD axions. Nevertheless, such searches ought be done, if for no other reason than to check our assumptions both about axion models, and inferential limits on the axion from cosmology or astrophysics. Nature bats last, and one should be open to surprise. Figure 6 shows two conceptions for experiments based on lasers and dipole magnets. Photon regeneration (a.k.a. "shining light through walls") relies on a symmetric arrangement of two such magnets; axions are coherently produced on one side of an optical barrier and reconverted to real photons on the other (Fig. 6, top) [15]. The probability to detect a photon per laser photon in is given by $\mathrm{P}(\mathrm{a} \rightarrow \gamma \rightarrow \mathrm{a})=\Pi^{2}$, where $\Pi=$ $(1 / 4)\left(\mathrm{g}_{\text {ary }} \mathrm{BL}\right)^{2}$; the $\mathrm{g}_{\mathrm{ary}}{ }^{4}$ dependence of the signal severely limits the sensitivity of the search, and this is a general feature of all such laboratory experiments. Utilizing two Colliding Beam Accelerator superconducting dipole magnets (4.4 $\mathrm{m}, 3.7 \mathrm{~T}$ ) at Brookhaven, Ruoso et al. set limits by photon regeneration, of $\mathrm{g}_{\text {ary }}<$ $7.7 \times 10^{-7} \mathrm{GeV}^{-1}$, for $\mathrm{m}_{\mathrm{a}}<10^{-3} \mathrm{eV}[16]$.

Axion-photon mixing can, in principle, also be observed through subtle changes in the polarization state of light in a magnetic field. In the mid-1980's, Maiani, Petronzio and Zavattini proposed an elegant measurement of higher-order QED in an optical cavity within a strong magnetic field (Fig. 6, bottom). The "light-bylight" Delbrück scattering diagram induces a miniscule birefringence to the vacuum, $\mathrm{n}_{11}>\mathrm{n}_{\perp}>1$, the splittings being proportional to $\left(\mathrm{B} / \mathrm{B}_{\text {crit }}\right)^{2}$, the Schwinger critical field being $\mathrm{B}_{\text {crit }}=\mathrm{m}^{2} / \mathrm{e} \approx 4.41 \times 10^{13}$ gauss. Thus light entering the cavity linearly polarized, e.g. at $45^{\circ}$ to the magnetic field, would exit 
elliptically polarized. They pointed out, however that fluctuations of the photon into a virtual axion also would contribute to the birefringence, the magnitude depending of course on the strength of coupling. Moreover, the production of real axions in the dipole magnet would result in a depletion of the parallelpolarized photons in the beam, but not for those perpendicularly polarized, resulting in a dichroism, or rotation of the plane of polarization, by an angle $\varepsilon=$ $(1 / 16)\left(\mathrm{g}_{\text {ary }} \mathrm{BL}\right)^{2}$ per pass. Both polarization observables, the ellipticity $\Psi$ and rotation $\varepsilon$, are linearly cumulative in the number of traversals $N$ through the magnet, putting a premium on the finesse of the Fabry-Perot. Semertzidis et al. at Brookhaven set limits on both effects in an optical cavity with $\mathrm{N} \sim 500$, within a $4.4 \mathrm{~m}, 5 \mathrm{~T}$ magnet (Figure 7, upper right) [17]. Unfortunately, the signal expected from higher-order QED was four orders of magnitude below their demonstrated sensitivity, and that associated with a standard QCD axion would have been another twelve orders of magnitude weaker yet. Recently, however the PVLAS collaboration at Legnaro reported a positive result with an improved apparatus ( $\mathrm{N} \sim 40,000$ at $1064 \mathrm{~nm}$, within a $1 \mathrm{~m}, 5 \mathrm{~T}$ magnet), measuring a polarization rotation of $(3.9 \pm 0.5) \times 10^{-12} \mathrm{rad} /$ pass (Figure 7 , lower right) [18]. If interpreted as the production of a light pseudoscalar, it is consistent with the earlier null result for $\mathrm{m}_{\mathrm{a}} \approx(1-1.5) \times 10^{-3} \mathrm{eV}$, and $\mathrm{g}_{\text {ayy }} \approx(1.6-5) \times 10^{-6} \mathrm{GeV}^{-1}$. While a whole litany of possible spurious origins has been excluded for the exceedingly small rotation, there are obvious unidentified systematic effects associated with the measurement. More problematically, the result is difficult to reconcile with the much stronger limits from Horizontal Branch stars, and the CAST solar experiment. A new photon regeneration experiment is being planned that would quickly be able to confirm or exclude the particle interpretation of PVLAS.

\section{Will the axion soon be found?}

Were a particle interpretation of the PVLAS results to be borne out, its implications for physics would be imponderable; it might have nothing at all to do with either the strong- $\mathrm{CP}$ or dark matter problems. In any case, one can 
expect the experimental question to be resolved in the near future. Should a more conventional explanation be found for the extraordinarily tiny effect, we venture that the resonant cavity experiments, betting on axionic dark matter, offer the best prospects for discovery the axion. Their sensitivity is already very nearly good enough, but additional improvements in noise temperature will still be required to speed up the scanning rate in mass, to provide definitive answers within five to ten years.

Axionic dark matter, should it discovered, would provide a rich quantum system to study. Its coherence length, measured in a two-cavity experiment, would be macroscopic even for the virialized component of the axion halo $(\lambda \sim 10-100 \mathrm{~m})$ and much longer for any fine structure component. More significantly, any nonthermalized structure could reveal important details of the history of our galactic formation.

The Laboratory Directed Research and Development program at LLNL is gratefully acknowledged for enabling technology development. This work was performed under the auspices of the U.S. Department of Energy, Contract No. W-7405-Eng-048. We acknowledge Larry Lasher, the Pioneer 10 project manager for the fascinating history and lore on its telemetry system.

This work was performed under the auspices of the U. S. Department of Energy by University of California, Lawrence Livermore National Laboratory under contract W-7405-Eng-48.

* ADMX is a collaboration of LLNL, U. Washington, U. Florida, U.C. Berkeley, and NRAO.

The authors are the co-spokespersons of the ADMX experiment. Leslie Rosenberg is Professor of Physics at the University of Washington, and Senior Scientist at Lawrence Livermore National Laboratory. Karl van Bibber is Deputy Director of the Laboratory Science and Technology Office at Lawrence Livermore National Laboratory. 


\section{References}

1. R. Peccei and H. Quinn, Phys. Rev. Lett. 38 (1977) 1440, and Phys. Rev. D16 (1977) 1791

2. S. Weinberg, Phys. Rev. Lett. 40 (1978) 223; F. Wilczek, ibid. 279

3. P. Sikivie, Physics Today 49 (1996) 22

4. P. Sikivie, Phys. Rev. Lett. 51 (1983) 1415, and Phys. Rev. D32 (1985) 2988

5. S. De Panfilis et al., Phys. Rev. Lett. 59 (1987) 839; W. Wuensch et al., Phys. Rev. D40 (1989) 5153

6. C. Hagmann et al., Phys. Rev. D42 (1990) 1297

7. R. Bradley et al., Rev. Mod. Phys. 75 (2003) 777

8. C. Hagmann et al., Phys. Rev. Lett. 80 (1998) 2043; S. Asztalos et al., Ap. J. Lett. 571 (2002) 27; S. Asztalos et al., Phys. Rev. D64 (2001) 092003

9. Mück, M. et al., Appl. Phys. Lett. 75 (1998) 3545; Mück, M. and J. Clarke, J. Appl. Phys. 88 (2000) 6910

10. Y. Kishimoto, Phys. Lett. A 303 (2002) 279; M. Tada, ibid., 285

11. M. Tada et al., Phys. Lett. A 349 (2006) 488

12. G.G. Raffelt, Stars as Laboratories for Fundamental Physics, University of Chicago Press, Chicago (1990)

13. K. Zioutas et al., Phys. Rev. Lett. 94 (2005) 121301

14. K. van Bibber, P.M. McIntyre, D.E. Morris, G.G. Raffelt, Phys. Rev. D 39 (1989) 2089

15. K. van Bibber, N.R. Dagdeviren, S.E. Koonin, A.K. Kerman, H.N. Nelson, Phys. Rev. Lett. 59 (1987) 759; see also A.A. Ansel'm, Yad. Fiz. 42 (1985) 1480 for a similar scheme for strictly massless pseudoscalars

16. G. Ruoso et al., Z. Phys. C (1992) 505

17. Y. Semertzidis et al., Phys. Rev. Lett. 64 (1990) 2988; R. Cameron et al., Phys. Rev. D 47 (1993) 3707

18. E. Zavattini et al., Phys. Rev. Lett. 96 (2006) 110406 


\section{Figure captions}

Figure 1. (Left) The cavity microwave experiment, with conventional microwave amplifier photon detection. The experiment consists of three basic components: a large-volume superconducting magnet, a high- $\mathrm{Q}$ tunable cavity, and an ultrasensitive microwave amplifier, constituting the front end of a double-heterodyne radio receiver. A power spectrum is calculated both with a resolution appropriate to the expected virialized axion signal $\left(\Delta \mathrm{E}_{\mathrm{a}} / \mathrm{m}_{\mathrm{a}} \sim 10^{-7}\right)$; but also with much higher resolution $\left(\sim 10^{-11}\right)$ to search for any fine structure in the axion signal due to predicted late-infall non-thermalized axions. (Right) The experimental tower just withdrawn from the superconducting magnet and cryostat (below floor level) of the Axion Dark-Matter eXperiment at LLNL.

Figure 2. Experimental limits of the Sikivie microwave cavity experiments (Rochester-Brookhaven-Fermilab [5], University of Florida [6], and ADMX [8]). The improvement resulted both from a significant scale-up of the experiment, and a roughly order-of-magnitude reduction in the noise temperature of gigahertz HFET amplifiers, to $\sim 1 \mathrm{~K}$ today.

Figure 3. (Left) The microstrip-coupled dc SQUID amplifier. By terminating the microstrip line with a variable capacitor, in situ tuning has been demonstrated over a factor of two in frequency. (Right) Electronic noise vs. physical temperature of a $700 \mathrm{MHz}$ microstrip-coupled SQUID amplifier. The intrinsic noise ultimately bottoms out at $\mathrm{T}_{\text {elec }} \sim 50 \mathrm{mK} \sim 1.5 \mathrm{~T}_{\text {quant }}$.

Figure 4. (Left) Schematic of the Rydberg atom single-quantum detector. (Right) No evidence for noise beyond thermal is seen down to $60 \mathrm{mK}$, due to the highly selective field ionization technique in the Kyoto experiment [11]. 
Figure 5. (Bottom) Photograph of the CAST experiment at CERN, utilizing a 10 meter long, 10 tesla LHC dipole to search for axions emitted from the Sun. (Insert) An x-ray optic consisting of nested grazing-incidence shells to focus the signal to a spot on a CCD, deployed on one of the magnet's four separate apertures. The other apertures are instrumented with Micromegas and TimeProjection Chamber (TPC) detectors. (Upper left) The spectrum of solar axions expected from the standard solar model. (Upper right) Region of mass-coupling space excluded by CAST, and previous axion helioscope searches [13]. Shown also is the expected sensitivity from the CAST Phase II run, where the magnet bores are filled with gas to increase the mass reach of the experiment.

Figure 6. Searches for axions configured on lasers and high energy accelerator dipole magnets. (Top) Photon regeneration, or "shining light through walls". (Bottom) Polarization effects associated with light traversing a strong magnetic field. Ordinary higher-order QED "light-by-light" scattering, and axion-photon mixing both contribute to vacuum birefringence, i.e. linearly polarized light becoming elliptically polarized. The production of real axions leads to vacuum dichroism, i.e. net rotation of the plane of polarization, due to the selective depletion of photons in the beam polarized parallel to the magnetic field.

Figure 7. (Left) Photograph of the PVLAS experiment. The superconducting dipole magnet is mounted vertically on a turntable and rotates about the axis of the Fabry-Perot cavity at $0.3 \mathrm{~Hz}$ to modulate the searched-for signal. (Right, upper) Exclusion region from the absence of vacuum dichroism in a magnetic field, from the experiment of Semertzidis et al. [17]. (Plotted is $\mathrm{M} \equiv \mathrm{g}_{\text {ary }}{ }^{-1}$.) The red symbol indicates the region of a positive signal reported by Zavattini et al. [18]. (Right, lower) PVLAS spectra with and without the magnet energized. The linearly polarized output of the Fabry-Perot is optically modulated at $506 \mathrm{~Hz}$ to produce a carrier signal. A real physical signal due to vacuum dichroism is indicated by the sidebands at $2 \omega$, due to the $\mathbf{E} \cdot \mathbf{B}$ nature of the axion-photon 
interaction, $\omega$ being the rotation frequency of the dipole magnet about the axis of the Fabry-Perot $(\omega=0.3 \mathrm{~Hz})$. The sidebands at $1 \omega$ are not understood, but are not correlated in phase with the $2 \omega$ signal. 

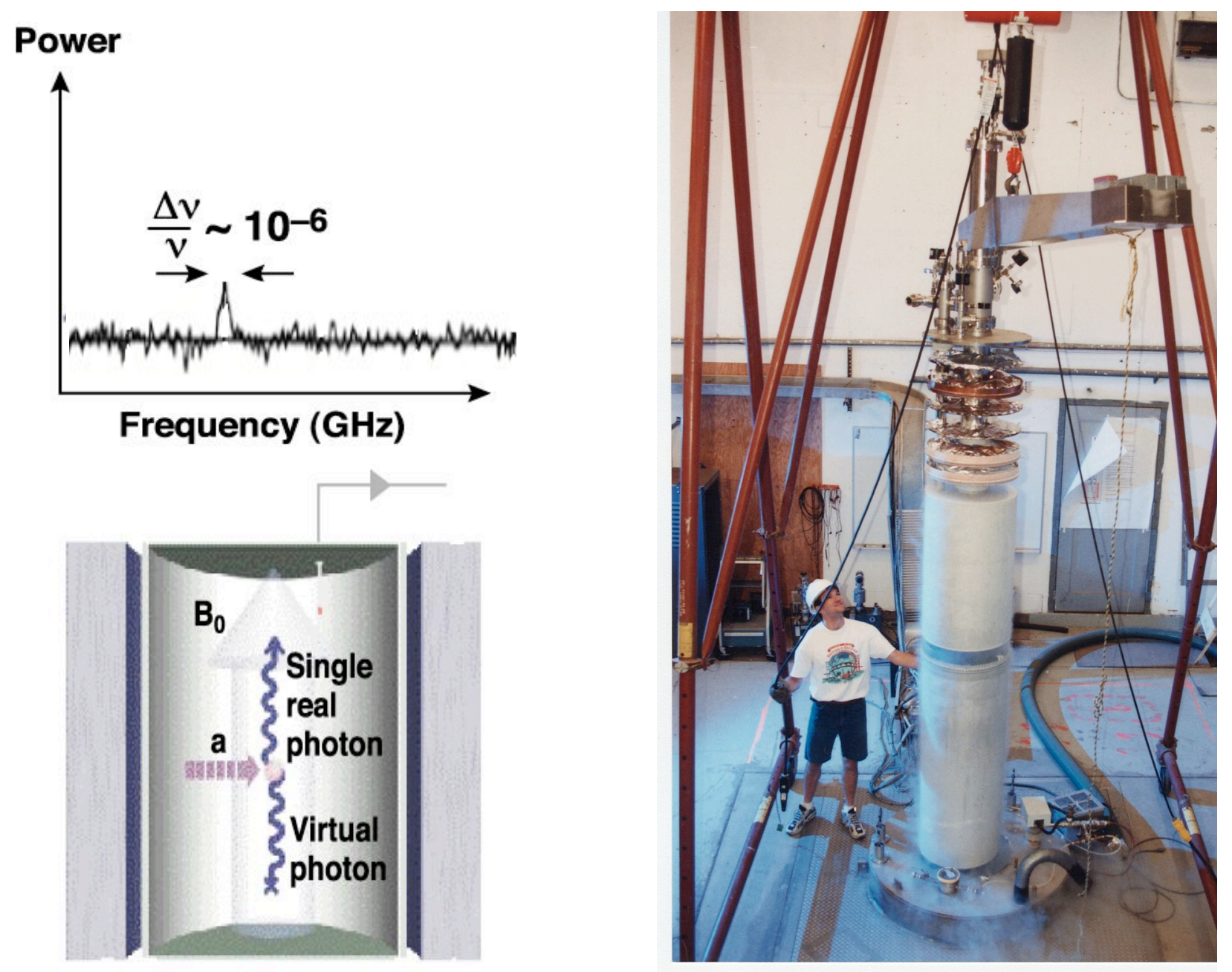

Figure 1 


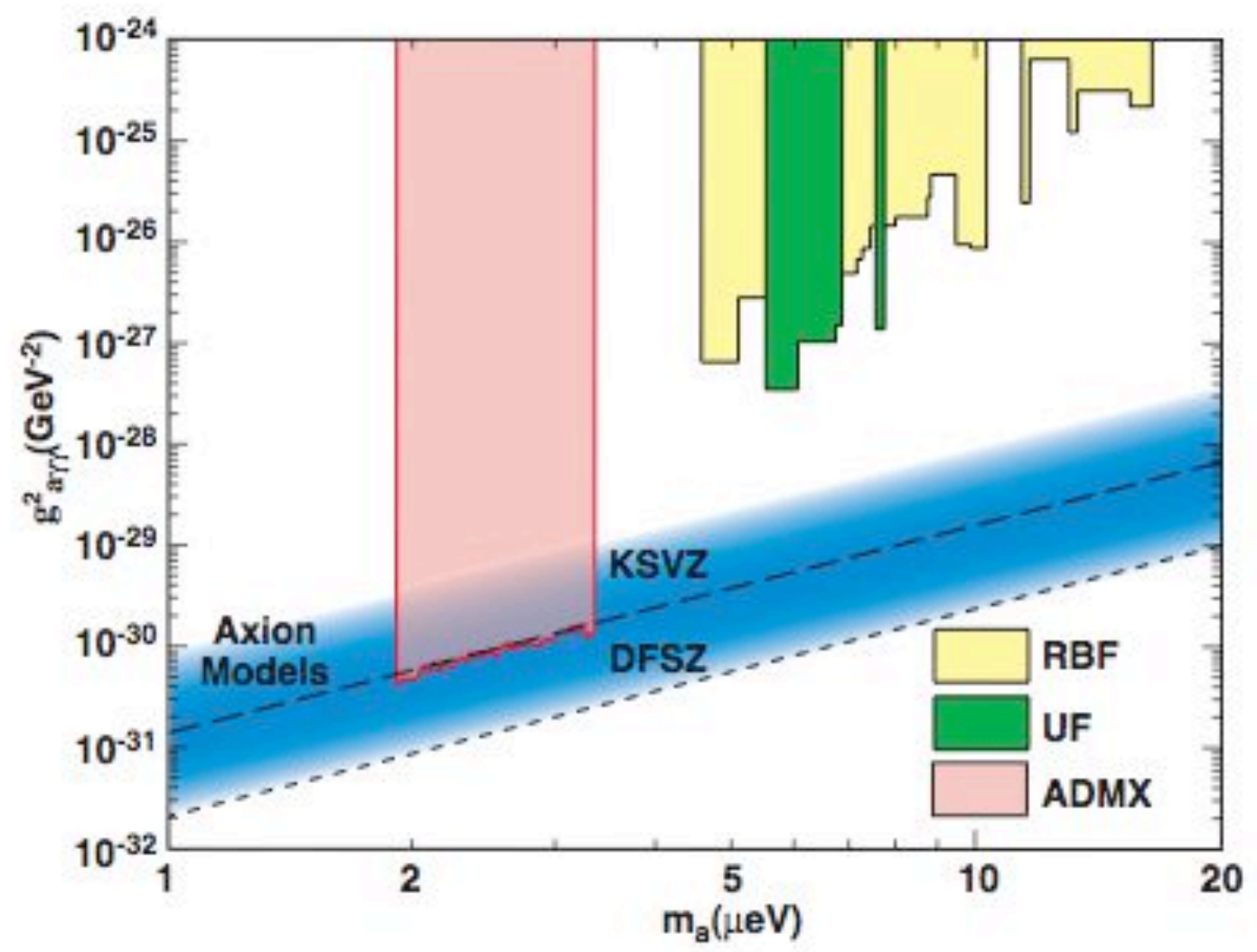

Figure 2 
Figure 3
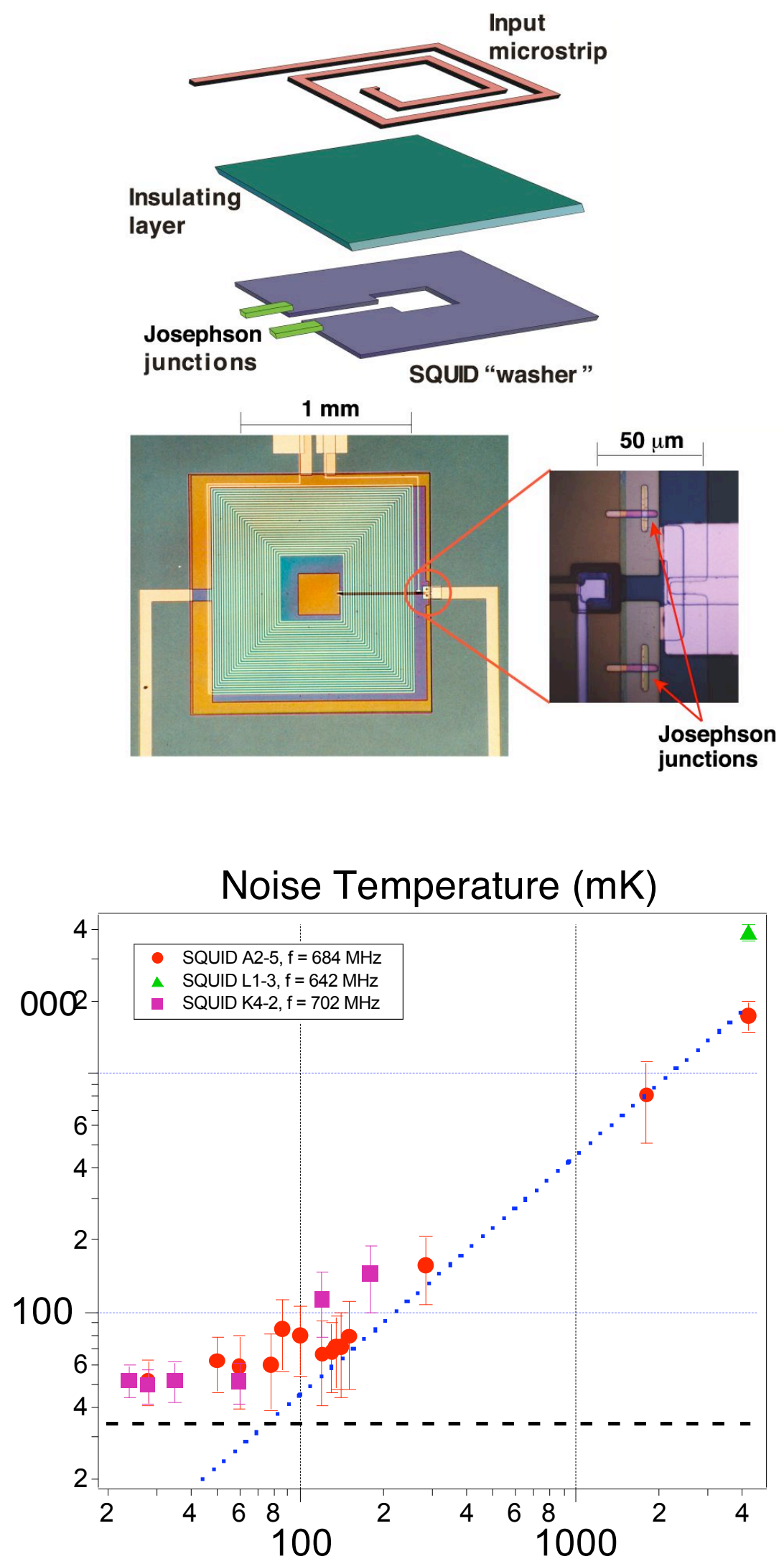

Physical Temperature (mK) 

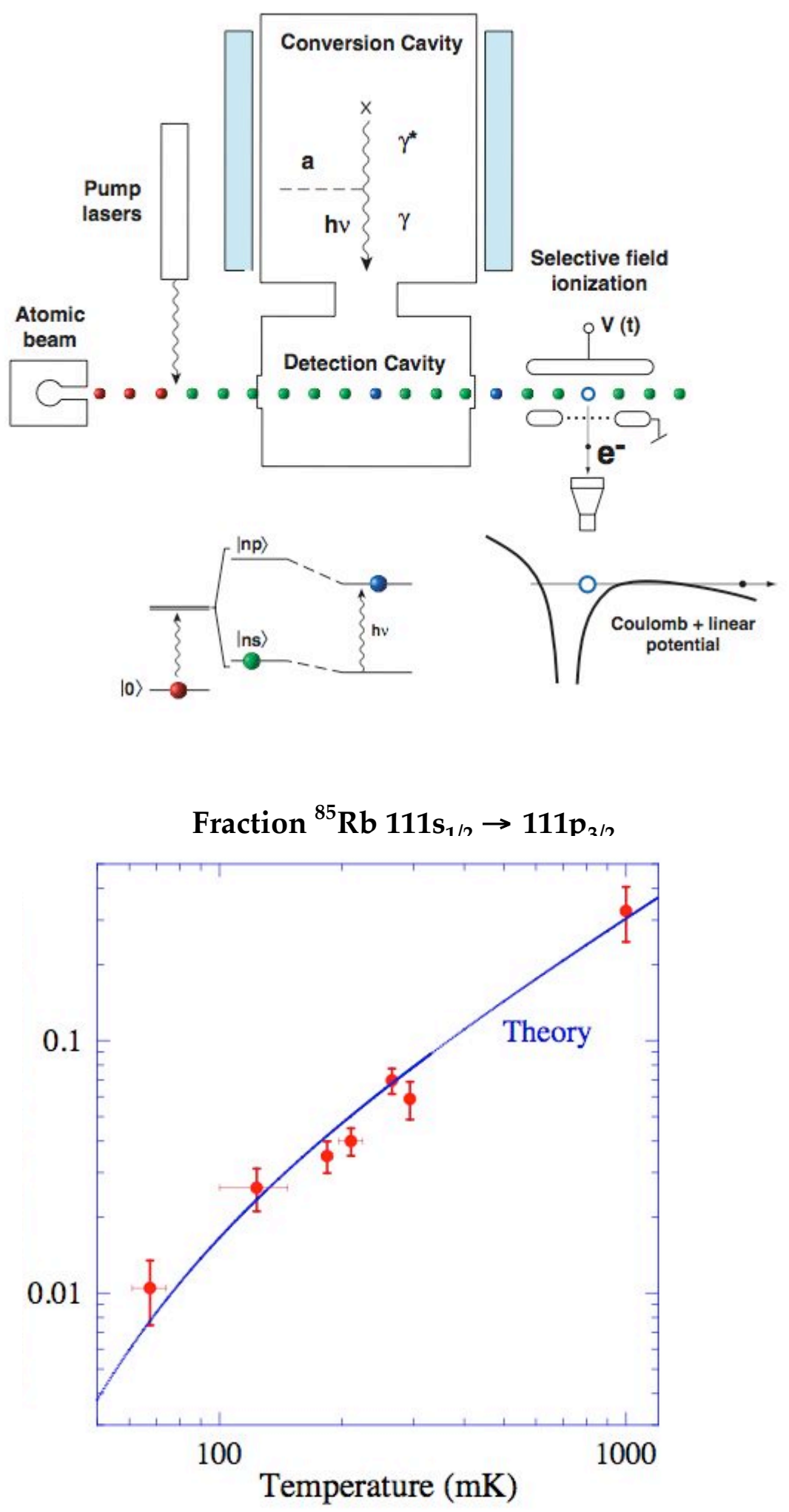

Figure 4 
Flux $\left[10^{10} \mathrm{~m}_{\mathrm{a}}(\mathrm{eV})^{2} \mathrm{~cm}^{-2} \mathrm{sec}^{-1} \mathrm{keV}^{-1}\right.$
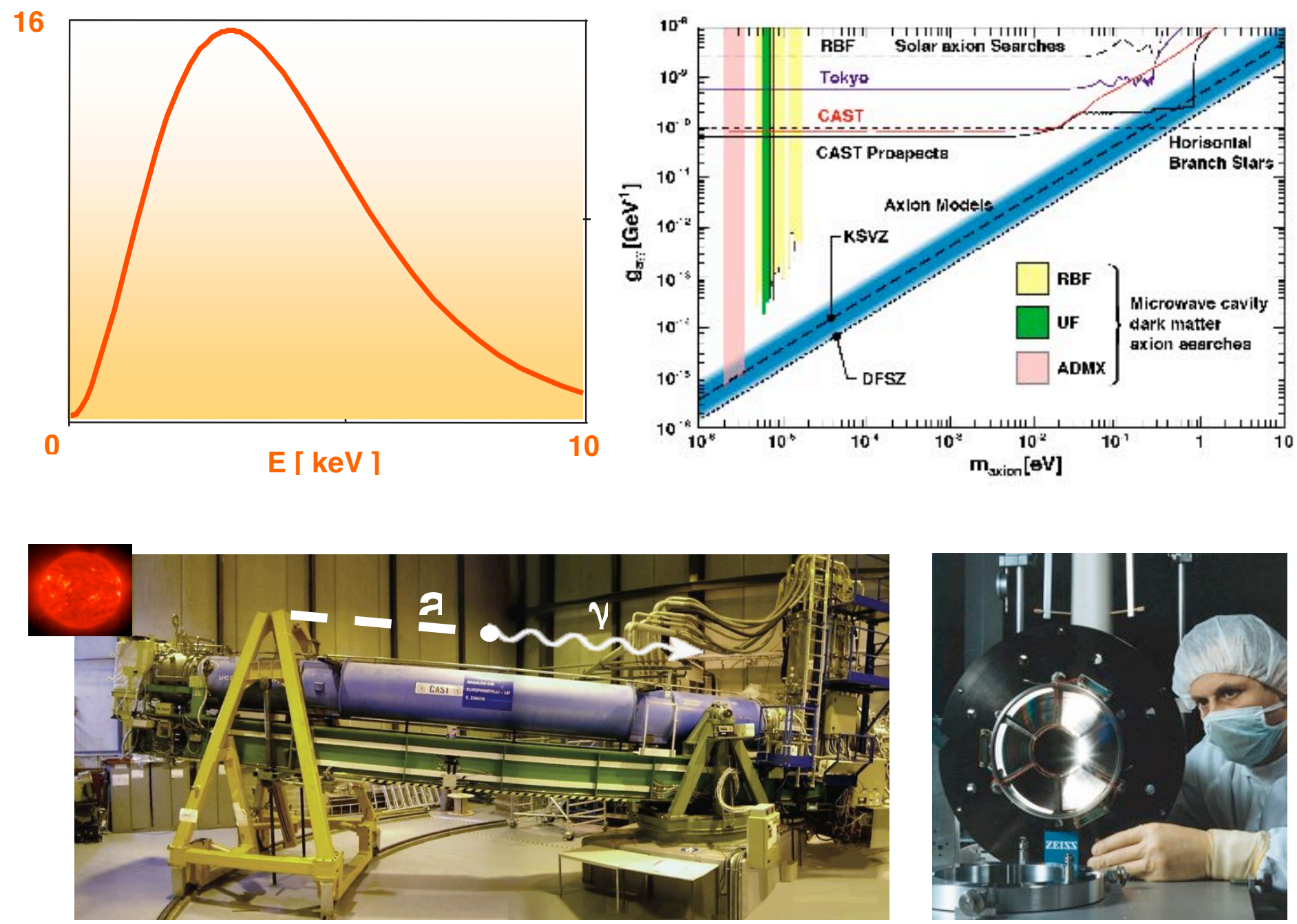

Figure 5 

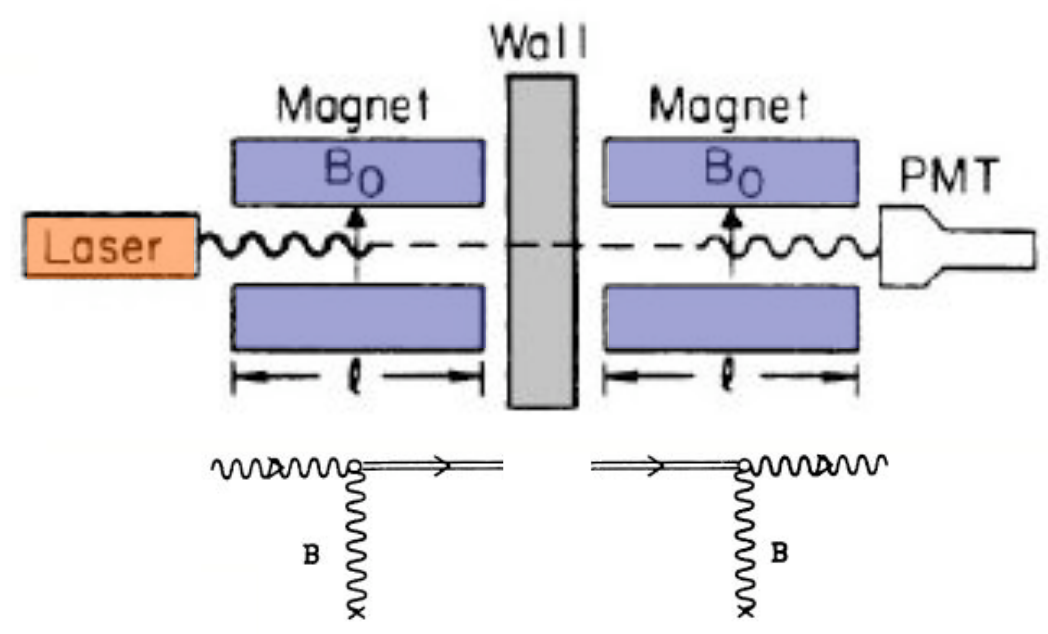

Fabry-Perot
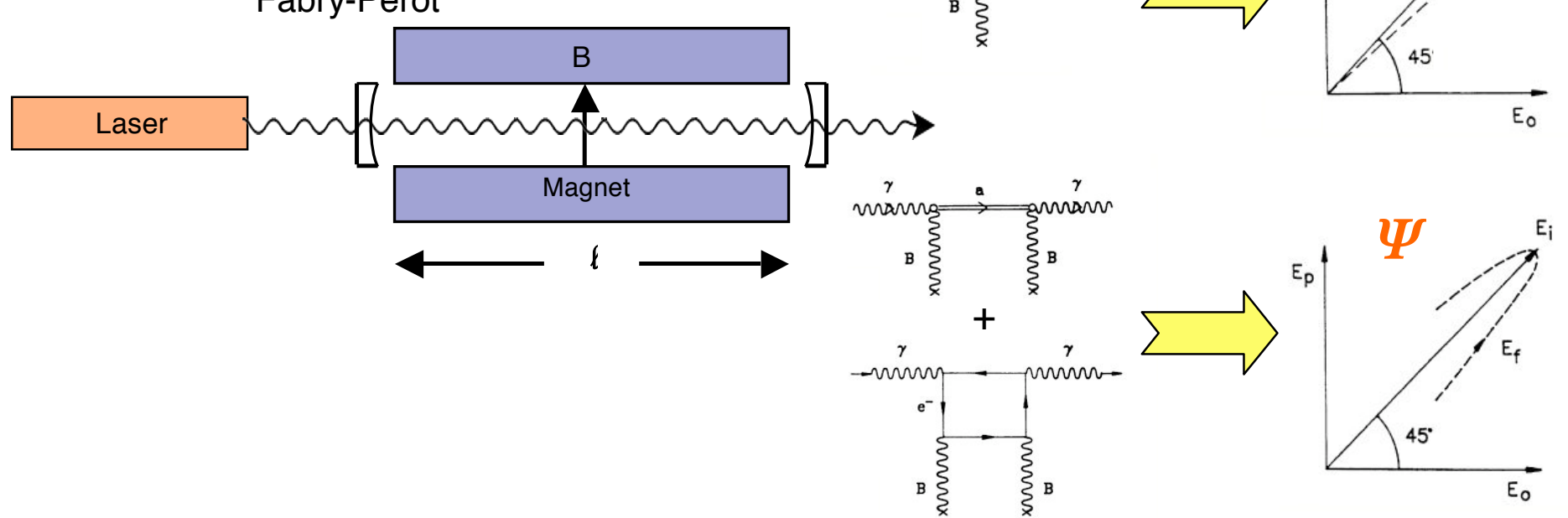

Figure 6 

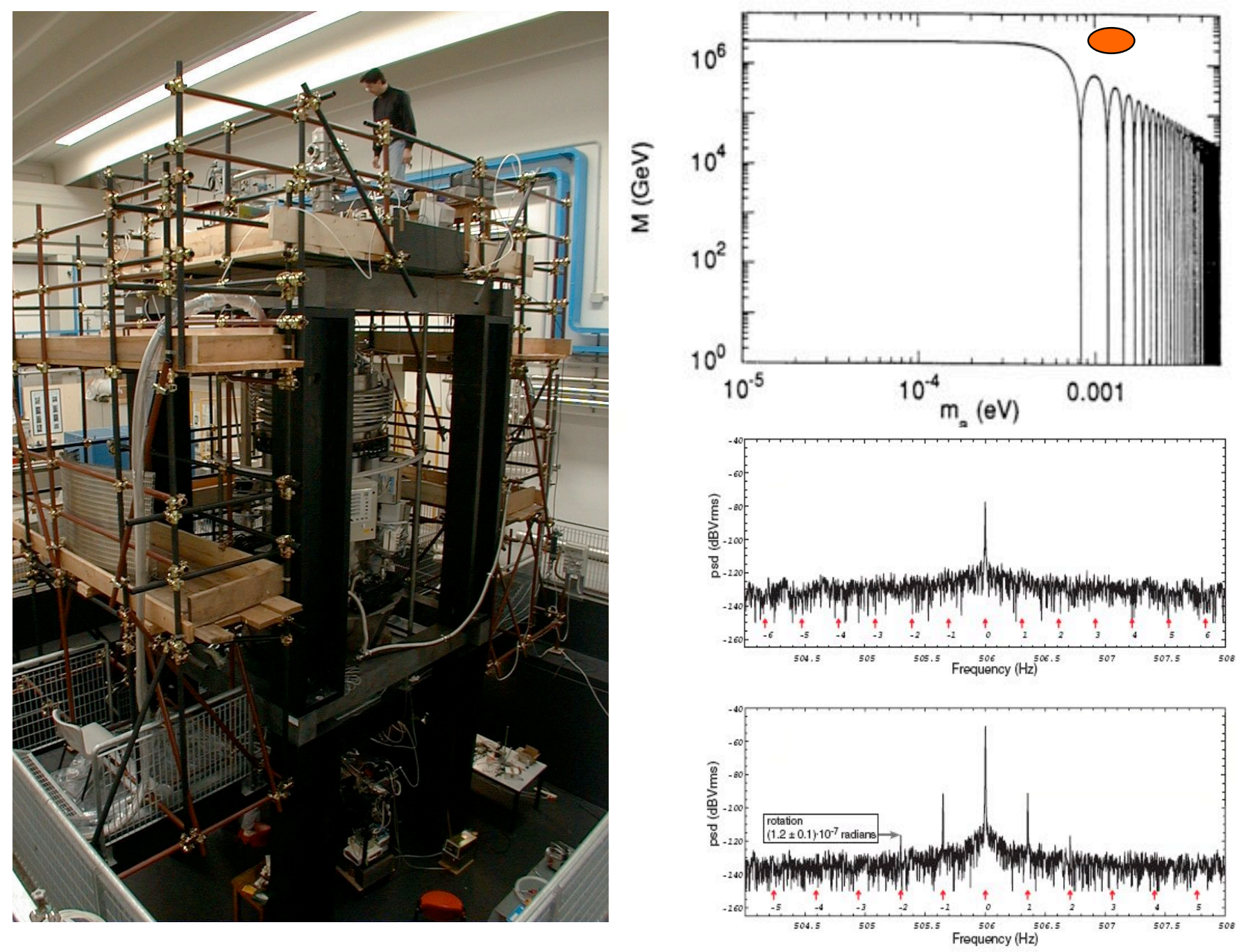

Figure 7 